female; 68.3 years (19.3)] were included. The median concentrations $(\mu \mathrm{g} / \mathrm{L})$ of iron, zinc and selenium were significantly lower in severe CDI cases compared with those with mild infection. Using CART decision tree analysis, 4 variables (peak WCC, selenium, Charlson co-morbidity index and magnesium) were found to be good predictors of severe CDI with sensitivity, specificity, accuracy, positive predictive value, negative predictive value and ROC of $84.8 \%, 98.9 \%, 94.3 \%, 97.5 \%$, $93.1 \%$ and 0.848 , respectively.

Conclusions Novel therapeutic interventions that modulate the availability of trace metals may substantially impact on disease outcomes in CDI. Mechanistic studies are required to establish the source of metals detected and to determine their relevance to oxidative stress, impaired immune response and the promotion of C. difficile bacterial virulence.

\section{PTU-049 RECENT COLORECTAL CANCER INCIDENCE TRENDS: IS OVERDIAGNOSIS A PROBLEM IN SCREENING?}

\footnotetext{
${ }^{1,2}$ Joanne Morling* ${ }^{*}{ }^{1}$ Caroline Chapman, ${ }^{1,2}$ Richard Logan. ${ }^{1}$ Bowel Cancer Screening Programme, Nottingham, UK; ${ }^{2}$ Division of Epidemiology and Public Health, University of Nottingham, Nottingham, UK
}

\subsection{6/gutjnl-2018-BSGAbstracts.390}

Introduction Colorectal cancer (CRC) screening using biennial gFOBT was introduced in England in September 2006. ${ }^{1}$ and by 2010 was being offered $>90 \%$ of $60-69$ year olds, rising to $>95 \%$ of $60-74$ year olds by 2014 . Overdiagnosis of cancer is a major concern of cancer screening programmes; in breast cancer it has been estimated that around 50\% of screen detected cancers might be due to overdiagnosis. This study seeks to examine the trends in CRC incidence and ascertain what impact screening has had on incidence.

Methods Data for the period 2001-16 was extracted from the ONS website (www.ons.gov.uk) and CRC incidence rates by 5 year age bands from age 45 calculated. CRC was defined according to the ICD 10th Revision codes C18 (colon) and C19/C20/C21 (rectum, recto-sigmoid and anus). Changes in incidence rates in age groups never offered screening were compared with those offered screening (age group 60-74 years).

Results As shown in table 1 CRC incidence in the 60-64 age band has increased, peaking at 22\% (M) and 17\% higher $(\mathrm{F})$ in $2009 / 10$ coinciding with the completion of screening rollout before reducing somewhat. In the 65-69 age band there were similar peaks $(21 \% \mathrm{M}$ and $17 \% \mathrm{~F})$ in $2008-10$ before a decline to below pre-screening rates. In the 70-74 age band for whom screening started in 2010 there were peaks in 2011/12 (17\% M and 19\% F) before declining below prescreening rates. These patterns were similar for both colon (C18) and for rectal (C19-21) cancers.

Abstract PTU-049 Table 1 Relative changes in colorectal cancer incidence (C18-21) by 5 year age-band in England \& Wales from 2005 to 2015

\begin{tabular}{llllllllll}
\hline & $45-$ & $50-54$ & $55-59$ & $60-64$ & $65-$ & $70-74$ & $75-79$ & $80-$ & $85+$ \\
& 49 & & & & 69 & & & 84 & \\
\hline Men & $+1 \%$ & $-5 \%$ & $+1 \%$ & $+12 \%$ & $-6 \%$ & $-11 \%$ & $-10 \%$ & $-3 \%$ & $+3 \%$ \\
\multirow{2}{*}{$\begin{array}{l}\text { Women } \\
\end{array}$} & $+15 \%$ & $+20 \%$ & $+18 \%$ & $+14 \%$ & $-4 \%$ & $-4 \%$ & $-4 \%$ & $+5 \%$ & $+8 \%$ \\
& $\%$ & & & & & & & & \\
\hline
\end{tabular}

Conclusion At this point we could find no evidence of over diagnosis of CRC. While there has been a $13 \%$ increase in CRC incidence in the 60-64 age band, consistent with the first (prevalent) screening round there has been no sustained increase in the older age bands offered screening. In contrast there has been an increase in incidence of rectal cancer in women under age 60 .

\section{REFERENCE}

1. Logan RF, Patnick J, et al. Outcomes of the BCSP in England after the first 1 million tests. GUT 2012 0ct;61:1439-46.

\section{PTU-050 THE SUCCESSFUL IMPLEMENTATION OF FAST-TRACK ROUTINE TESTING FOR MICROSATELLITE INSTABILITY IN A COLORECTAL CANCER PATHWAY}

${ }^{1}$ Wee Sing Ngu*, ${ }^{1}$ Sarah Mills, ${ }^{2}$ Harsh Sheth*, ${ }^{2}$ John Burn, ${ }^{1}$ Thomas Lee. ${ }^{1}$ Northumbria Healthcare NHS Foundation Trust, Newcastle Upon Tyne, UK; ${ }^{2}$ Institute of Genetic Medicine, Newcastle University, Newcastle upon Tyne, UK

\subsection{6/gutjnl-2018-BSGAbstracts.391}

Introduction The National Institute for Health and Care guidelines (DG27, Feb 2017) recommend that all patients with colorectal cancer (CRC) should undergo testing for deficient deoxyribonucleic acid (DNA) mismatch repair activity, whose by-product is microsatellite instability (MSI) in DNA. Historically in our trust, MSI testing was done infrequently, in selected high-risk patients, on preserved pathology specimens and with a long wait for results. A new patient care pathway incorporating MSI testing on fresh biopsy tissue with a rapid turnaround time was introduced in January 2017. This service evaluation reviewed performance in the first year of this new pathway.

Methods Endoscopists were asked to send an additional fresh biopsy for MSI assay at endoscopic diagnosis of significant neoplasia from January 2017. Data for all patients newly diagnosed with CRC between 1 st Jan 2017 to 31 st December 2017 were exported from a prospectively populated database. Results A total of 374 patients were identified, median age 72 (range 30-96) of whom 226 (60.4\%) patients were diagnosed at endoscopy. One hundred and ninety-one $(51.1 \%)$ of all patients had MSI assays performed, 142 (62.8\%) of those endoscopically diagnosed. Twelve (6.3\%) of the patients tested were MSI-high. Median time from submission of sample to result was 13 days (range 3-32).

Conclusions Compliance with MSI testing at endoscopic diagnosis is not yet $100 \%$, but this study illustrates that the MSI test can be integrated into the patient care pathway in an NHS setting and used to personalise patient care as turn-around times are sufficiently short for the results to be integrated into pre and post-operative multidisciplinary team meeting discussions.

\section{PTU-051 SHOULD FOBT POSITIVE PATIENTS WITH PREVIOUS LOW RISK IN SCREENING PROGRAM COLONOSCOPY HAVE FURTHER COLONOSCOPY?}

${ }^{1}$ David Nylander*, ${ }^{2}$ Emma Crossland, ${ }^{2}$ Heather Dixon. 'Newcastle Upon Tyne NHS Foundation Trust, Newcastle upon Tyne, UK; ${ }^{2}$ North of Tyne Bowel Cancer Screening Centre, Newcastle upon Tyne, UK

\subsection{6/gutjnl-2018-BSGAbstracts.392}

Introduction and aim Current British Society of Gastroenterology guidelines suggest patients who are deemed low risk after 